\title{
DESALINATION
}

ELSEVIER

Desalination 103 (1995) 233-247

\section{Pore formation mechanism of membranes from phase inversion process}

\author{
Tai-Horng Young ${ }^{\mathrm{a}^{*}}$, Leo-Wang Chen ${ }^{\mathrm{b}}$ \\ ${ }^{a}$ Center for Biomedical Engineering, College of Medicine, National Taiwan University, Taipei 10016, Taiwan, ROC \\ ${ }^{b}$ Department of Chemical Engineering, National Taiwan University, Taipei 10764, Taiwan, ROC
}

Received 6 July 1994; accepted 28 March 1995

\begin{abstract}
The formation of pores in membranes obtained by phase inversion is discussed on the basis of thermodynamic and diffusional aspects of phase separation processes. We demonstrated that different types of phase separation are responsible for the formation of the porous structure in ethylene-vinyl alcohol (EVAL) membranes. Special attention is paid here to the formation mechanism of the finger-like cavities. The results are consistent with observations of pore formation occurring on wet-casting membrane formation process and provide a greater understanding of the formation mechanism of pores of casting membranes.
\end{abstract}

Keywords: Liquid-liquid phase separation; Nucleus; Diffusion; Top layer

\section{Introduction}

Since the development of asymmetric type membranes by Loeb and Sourirajan [1], the field of membrane science and technology has experienced increasing growth. Asymmetrical membranes consist of a thin top layer supported by a porous sublayer and two structures can be obtained in the sublayer; one is membranes with spongelike structure and the other with large finger-like

"Corresponding author. pores (macrovoids). The presence of macrovoids is not generally favorable because they may lead to weak spots in the membrane.

Asymmetric membranes are generally prepared by the phase inversion method [2]. In this process a casting solution consisting of polymer and solvent is immersed into a nonsolvent coagulation bath. Interchange of solvent and nonsolvent due to diffusion causes the casting solution to go through a phase transition by which the membrane is formed. The development of the phase inversion technique has preceded many systematic studies of 
the effects of relevant parameters and the mechanism of membrane formation [3-17]. Membrane structure, especially pore size and its distribution, can be controlled for each specific application depending on the choice of the polymer, solvent, nonsolvent and preparation parameters.

Clearly the principal pore-forming processes occurring in the phase inversion process contain both the thermodynamics of the ternary system and mass transfer properties. Thermodynamics should explain the effects of different ternary systems on the construction of a phase diagram. Altena and Smolders [18] and Yilmaz and McHugh [19] have published relatively complete calculations of the ternary system based on the Fiory-Huggins theory to analyze the behavior of the phase diagram. The mass transfer property mainly involves the relative diffusion rates of solvents and nonsolvents. Some investigators have carried out different models to describe the phenomena of mass transfer of membrane formation [13,17,20-25]. Despite these extensive research efforts, the actual formation mechanism of pores and specifically the growth of pores is not well understood. We feel that it would be of great value for the improvement of membrane preparation processes if our knowledge of pore formation phenomena could be clearly understood. Thus, this study is concerned with pore formation from small pores to finger-like pores by combining the thermodynamic concept of the phase diagram and relative diffusion rates of solvents and nonsolvents. Changes were made in the membrane preparation parameters in order to improve our understanding and control the formation process of pores. In particular, the concept of the driving force between solvents and nonsolvents was applied to predict the growth of pores in the different membrane structures.

\section{Theory}

The main route of the phase inversion process involves two different types of phase transition [12], which can be illustrated in a ternary phase diagram (see Fig. 1). These are: (1) liquid-liquid

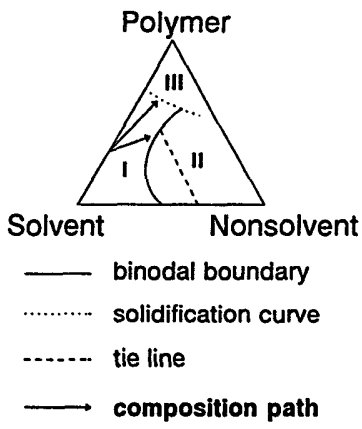

Fig. 1. Ternary phase diagram of EVAL-DMSO-water system. I: homogeneous solution region; II: two-phase region; III: solidification region.

phase separation, in which the completely miscible solution crosses the binodal boundary to enter the two-phase region (from I to II), and (2) solidification (from I to III or from II to III); since the viscosity of the polymer solution increases to a certain assumed value, the motion of polymer chains will be limited and the system can be regarded as a solid to fix the membrane structure.

\subsection{Liquid-liquid phase separation}

Liquid-liquid phase separation in miscible polymer solution can be caused by variation in temperature and/or composition of the mixture. When a homogeneous solution becomes thermodynamically unstable, the solution can decrease its free energy from mixing by dividing into two liquid phases of different composition, i.e., a nucleus of the polymer-poor phase that forms the nascent pore and a polymer-rich phase that surrounds the pore. Hence, a nascent pore comprises the polymer-poor phase surrounded by the polymer-rich phase. The two liquid phases are in thermodynamic equilibrium and can be connected by the tie line in the phase diagram.

There are not many experimental data on the location of the liquid-liquid demixing gap in terna- 
ry systems. Only for a few ternary systems are experimental data on binary interaction available. Cohen and coworkers [20] and Altena and coworkers [18] have calculated binodals and tie lines for some ternary systems. Some clear observations concerning the thermodynamic properties of the ternary system are:

1. The polymer concentration in the polymerpoor phase rapidly approaches a very small value in many circumstances. However, the polymer concentration in the polymer-rich phase is much higher at low mutual affinity between the solvent and the nonsolvent. Therefore, the polymer-rich phase will slow down the solidification rate, and the pore wall will have more time to grow at high affinity between the solvent and the nonsolvent.

2. The mutual affinity between the solvent and the nonsolvent has an effect on the position of the binodal curve. It appears that the high mutual affinity favors the demixing of the ternary system, i.e., the tendency of the location of the miscibility gap is to move towards the polymer-solvent axis in the ternary diagram. Consequently, less nonsolvent is needed for liquid-liquid phase separation.

3. The low end point of the tie line shifts towards the solvent corner of the ternary diagram when the mutual affinity between the solvent and the nonsolvent increases. This means the volume fraction of nonsolvent in the polymer-poor phase requires only small amounts. Therefore, a higher mutual affinity decreases the amount of nonsolvent necessary for pore formation.

\subsection{Diffusion-controlled model}

To predict which type of phase transition will occur in the membrane formation process, we must know the composition variation of the casting solution. However, the composition variation of a ternary system is very complex. For simplification, in our previous model [13] we divided the casting solution into $N$ hypothetical inner layers parallel to the surface of the casting solution and carried out the mass balance to determine the local composition variation in each layer. We treated the membrane formation process as a one-dimension process and a pseudobinary system (the perpendicular movement of the polymer is neglected). Some assumptions can be seen in Ref. [13]. The most important assumption is that if the thickness of each layer is small enough, the diffusion ratio of solvent to nonsolvent can be considered as a constant value for each layer. The ratio can been written as

$k=\frac{\tilde{n}_{2}}{\bar{n}_{1}}$

$\tilde{n}_{1}$ and $\tilde{n}_{2}$ are the diffusion flux of nonsolvent and solvent, respectively. In this case the continuity equations and diffusion equations of each layer can be solved to get, after some manipulation, the following result for the local composition variation:

$$
\phi_{1}=\frac{r-\bar{\rho}_{0}^{\prime} / \rho_{1}^{0}}{r-k}+\frac{r \phi_{3}}{k-r}
$$

In this equation $r$ represents the density ratio of solvent to nonsolvert, $\rho_{1}^{0}$ is the density of nonsolvent, and $\bar{\rho}_{0}^{\prime}$ is shorthand for the quantity $\left[1+(k-1) \bar{W}_{10}\right] \bar{\rho}_{0}$, where $\bar{W}_{10}$ and $\bar{\rho}_{0}$ are the initial nonsolvent weight fraction of the casting solution and the initial casting solution density both on a polymer-free basis. Since all the above mentioned parameters are constant, Eq. (2) is a linear equation for $\phi_{1}$ (the volume fraction of nonsolvent) and $\phi_{3}$ (the volume fraction of polymer) at a fixed $k$ value. Therefore, the composition variation of each layer will be a straight line in the ternary phase diagram (see Fig. 1).

In order to apply Eq. (2), one must know the $k$ value, i.e., the diffusion ratio of solvent to nonsolvent. We found that the equilibrium amount of nonsolvent absorbed in dense membranes and the heat of mixing of nonsolvent and solvent could be used to predict the diffusion ratio [14]. The equilibrium amount absorbed represents the interaction between polymer and nonsolvent, on one hand, and the tendency of diffusion of nonsolvent into 
the casting solution, on the other. In the same way the heat of mixing of solvent and nonsolvent represents the interaction between solvent and nonsolvent, on one hand, and the diffusion tendency of solvent into the coagulation bath, on the other.

\subsection{Two-step mechanism model}

Although the equilibrium amount and the heat of mixing can predict the variation tendency of the diffusion ratio of solvent to nonsolvent, the determination of the flux ratio of each layer is a problem without an easy solution. It has been suggested that the membrane formation process can be considered as a two-step mechanism model [12]. The model can simplify the problem in two special cases: asymmetric membranes and homogeneous membranes.

When the $k$ value of the top layer is very large, namely, the solvent diffuses out rapidly from the surface casting solution, then the top layer is very dense. In the ternary phase diagram the composition path of the top layer directly enters the solidification region. All the $k$ values from the second layer to the bottom layer are influenced by the top layer since the dense top layer will increase the barrier for diffusion of solvent from and nonsolvent into the interior casting solution. Therefore, the dense top layer controls the diffusion ratio of solvent to nonsolvent and the composition path of the second layer to the bottom layer. Thus, the area from the second layer to the bottom layer is reduced to one sublayer. If the top layer only limits the diffusion of solvent, then the $k$ value of the sublayer is decreased and the composition path of the sublayer crosses the binodal to cause liquidliquid phase separation to form the porous structure. Consequently, an asymmetric membrane with a thin dense skin supported by a thick porous sublayer is formed.

On the other hand, if the top layer only limits the diffusion of nonsolvent, then the $k$ value of the sublayer is also large like that of the top layer and the composition path of the sublayer enters the solidification region to form the dense structure. In this case the $k$ value of each layer is approximately equal so that a homogeneous membrane is exhibited with each layer having the same composition path and dense structure.

When the $k$ value of the top layer is not large enough, namely, the solvent diffuses out slowly or nonsolvent diffuses into the casting solution rapidly, the composition path of the top layer may cross the binodal to cause liquid-liquid phase separation to form a porous top layer. The following diffusion rate of the sublayer is only slightly affected by the top layer because of its porous structure. As a consequence, the phase transition in the sublayer is the same as that in the top layer to form a homogeneous porous membrane.

Therefore, the two-step mechanism model depicts that the membrane consists of two layers: a top layer and a sublayer. The top layer is formed first at the casting solution-nonsolvent interface. The ratio of nonsolvent inflow to solvent outflow is of the utmost importance for the top layer structure. The structure of the sublayer depends on the local composition at the instant of phase transition, but it is directly influenced by the degree of aggregation of the polymer molecules at the top layer. If the sublayer structure is the same as the top layer structure (porous or dense), then the two-step mechanism is reduced to a one-step mechanism.

\subsection{Pore formation mechanism}

In the two-step mechanism model a dense skin is formed because solvent in the casting solution desolvates rapidly into the coagulation bath, and relatively little nonsolvent diffuses into the casting solution at the moment the casting solution and nonsolvent come into contact. The thickness of the skin layer will increase gradually until the diffusion of solvent from the sublayer solution through the dense skin layer into the coagulation bath is restrained. As a result, the composition of the sublayer does not change as rapidly, and the solution is within the initial homogeneous solution region. In the meantime nonsolvent diffuses through the dense top layer. When a certain 
Coagulation Bath

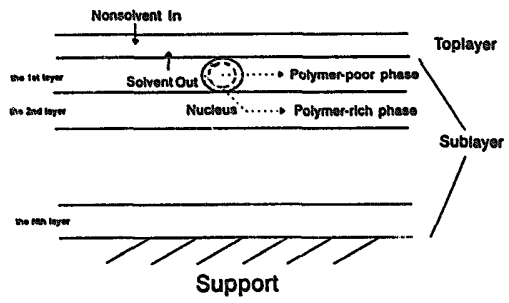

Fig. 2. Schematic representation of the casting solution layer and pore structure.

amount of nonsolvent has caused the composition variation to cross the binodal boundary to become unstable somewhere, the first nucleus of the polymer-poor phase occurs to form the nascent pore in the first layer of the sublayer (see Fig. 2). When more nonsolvent enters the different sites of the first layer, more pores occur.

A nascent pore will grow if nonsolvent can continually diffuse into it to induce solvent surrounding the nascent pore to diffuse into it. The diffusion of solvent into the growing pore will cause an increase in the polymer concentration of the polymer-rich phase surrounding the pore. When the surrounding polymer-rich phase enters the solidification region, a pore stops their growth; i.e., the solidification is the major factor determining pore wall structure. According to the first observation mentioned in liquid-liquid phase separation, the polymer concentration of the polymerrich phase will increase very greatly at low mutual affinity between solvent and nonsolvent so that a pore wall will immediately reach solidification. Furthermore, when the affinity between solvent and nonsolvent is relatively low, the diffusion process will be quite slow so as to result in the slow growing rate of the pore; the pore size is therefore comparatively small.

If a certain amount of coalescence of the polymer-poor phase occurs before the wall solidifies, open pores will occur (see Fig. 3). It has the tendency that small pores combine to a larger pore.

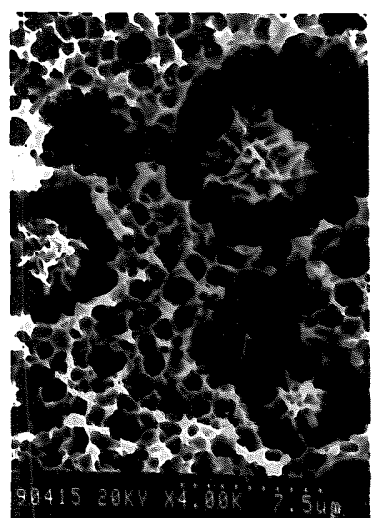

Fig. 3. EVAL membrane with typical open pores.

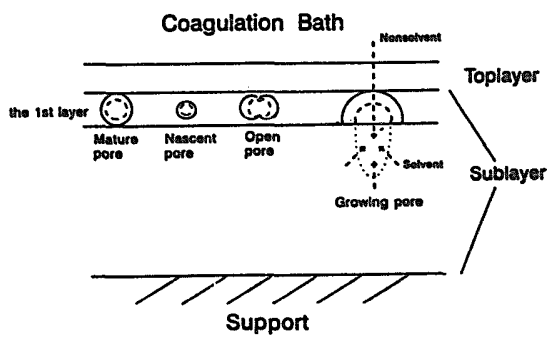

Fig. 4. Schematic diagram of pore formation and growth.

Because the induction times before any nuclei appear are different, the first layer of the sublayer has mature pores (pore wall has solidified), cpen pores, late forming nascent pores or growing pores when nonsolvent starts to enter the second layer (see Fig. 4). For a growing pore the upper peripheral solution may have solidified in the first layer, but the lower part of the pore does not solidify yet and may extend to the second layer. Therefore, the second layer has freshly forming pores or old pores formed in the first layer. In this way all the sublayer solutions will proceed the similar phase 
transition layer by layer due to the diffusion of solvent and nonsolvent to result in the generation of a membrane.

\subsection{Finger-like structure and sponge-like structure}

Clearly the polymer-poor phase contains solvent and nonsolvent. Thus, in a system with a large driving force for nonsolvents and solvents, only a little new nonsolvent into a pore is sufficient to introduce more solvent being depleted from the polymer solution surrounding the pore to maintain the equilibrium request of the polymerpoor phase. Since polymer molecules are clustered together to exclude solvent from within their domains, a small pore rapidly grows into a large pore. The concentration of the solution that surrounds the pore continually increases until it enters the solidification region to form the pore wall. In addition, at high affinity between solvent and nonsolvent, the pore wall solidifies late so it has adequate time to grow or even to form macrovoids in the ultimate membrane. The above conditions are advantageous to a finger-like structure formation. Therefore, finger-like structure formation requires that both solvent and nonsolvent can rapidly and consecutively diffuse into a ycunger pore before the solidification of the pore wall. The outcome is consistent with the trends observed in the liquid-liquid phase separation.

From the point of thermodynamics, the main driving force for the growth of the pores is the decrease in free energy on mixing solvent and nonsolvent in the pores. Therefore, the pores stop growing when solvent stops suppiying the growth of the pore. So the pores can grow to finger-like structures as solvents continually supply the growth of pores. Hence, membranes with the typical finger-like structures have a bottom skin layer shown in Fig. 5. This bottom layer is caused by the solidification in the last casting fluid since most solvent has been depleted thus stopping the supply of solvent.

The rate for the liquid-liquid phase separation is essentially dependent on nonsolvent content.

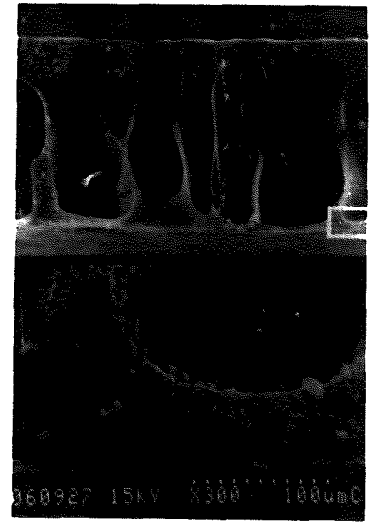

Fig. 5. EVAL membrane with finger-like structures and a typical bottom layer.

Hence, phase transition is delayed if nonsolvent cannot permeate appreciably into the casting solution to reach the binodal boundary. That is to say, the smaller the mutual affinity of solvent and nonsolvent, the more nonsolvent in the polymerpoor phase and the slower the mixing tendency of solvent and nonsolvent, the more induction time it takes for the nonsolvent into the casting solution to form nuclei, and the slower the liquid-liquid phase separation of the system. Thus nucleation starts until a certain amount of nonsolvent has diffused into the casting solution. Consequently, many nuclei are initiated later but at the same time. Hence, the growth of every nucleus will be limited by other nearby nuclei because every nucleus consumes solvent. In this way the growth of macrovoids is impossible, and only smaller pores are formed, i.e., a sponge-like structure is formed (see Fig. 6).

It is necessary for the finger-like structure that has a skin layer to limit the large amount of nonsolvent into the sublayer to induce many nuclei formations. However, if the skin layer cannot strictly limit the diffusion of nonsolvent or the 

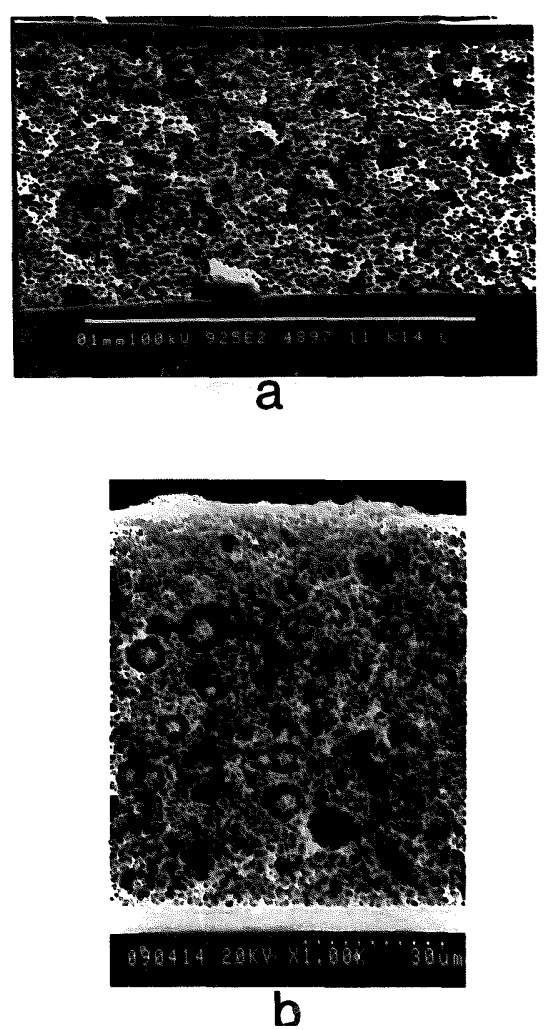

Fig. 6. Typical sponge-like membranes with (a) and without (b) a skin layer.

driving force between solvent and nonsolvent is not large enough, then it also does not form the finger-like structure. Therefore, the sponge structure is possible whether the skin layer is present or not (see Fig. 6).
Clearly within a finger the exchange of solvent and nonsolvent is very fast. Broens and coworkers [7] observed that the macrovoids move inward faster than the diffusion-controlled coagulation front. The liquid-liquid phase separation, therefore, occurs much slowly, and many smaller pores occur between fingers. Furthermore, the wall of the macrovoid does not solidify immediately during its growth so the liquid-liquid phase separation might take place surrounding the voids to form small pores (see Fig. 5).

\section{Experimental}

\subsection{Materials}

The ethylene-vinyl alcohoi (EVAL) copolymer with a content of $56 \mathrm{~mol} \%$ of vinyl alcohol monomeric units was obtained using previously reported techniques [9]. De-ionized and ultrafiltrated water was used in our experiments. Other reagents were of chemical reagent grade and were used without further purification.

\subsection{Membrane preparation}

The EVAL copolymers were dissolved in a different composition of solvent mixture to form a $25 \%$ polymer solution. The solution was spread on glass plates in a uniform thickness of about $175 \mu \mathrm{m}$. The glass plates were immersed immediately into the coagulation bath of varying nature of composition of nonsolvent. No evaporation step was performed before the casting solution was immersed into the coagulation bath unless otherwise reported. After polymers were precipitated, the membrane was removed from the glass plate and kept in a pure water bath. The morphology of the membranes was examined using a scanning electron microscope (SEM). Table 1 is a summary of the experimental data. The specific conditions for each membrane are included. The goal of the experiment was to check the theory described above. 
Table 1

Summary of experimental data

\begin{tabular}{lllll}
\hline Membrane & $\begin{array}{l}\text { Solvent } \\
\text { composition }\end{array}$ & $\begin{array}{l}\text { Nonsolvent } \\
\text { composition }\end{array}$ & $\begin{array}{l}\text { Casting solution } \\
\text { temperature }\left({ }^{\circ} \mathrm{C}\right)\end{array}$ & $\begin{array}{l}\text { Coagulation bath } \\
\text { temperature }\left({ }^{\circ} \mathrm{C}\right)\end{array}$ \\
\hline 1 & Pure DMSO & Pure water & 60 & 25 \\
2 & Pure DMSO & $67 \%$ DMSO, 33\% water & 60 & 25 \\
3 & Pure DMSO & 1st: 67\% DMSO, 33\% water & 60 & 1st: 25 \\
& & 2nd: pure water & 60 & 2 nd: 60 \\
4 & 80\% DMSO, 20\% water & Pure water & 60 & 25 \\
5 & 80\% DMSO, 20\% water & Pure water & 60 & 25 \\
6 & 60\% DMSO, 40\% water & Pure water & 70 & 25 \\
7 & 60\% IPA, 40\% water & Pure water & 60 & 25 \\
8 & 60\% IPA, 40\% water & Pure water & & 0 \\
\hline
\end{tabular}

\section{Results and discussion}

\subsection{Membrane 1, 2, 3}

The mechanism for the formation of pores outlined above allows many of the variables of the membrane preparation procedure to be rationalized. The interaction between solvent and nonsolvent is apparently a particularly important parameter. Using water as a nonsolvent, membrane 1 shows a dense skin with a finger-like structure (see Fig. 7). Due to the strong interaction between DMSO and water, DMíSO in the casting soiution desolvates rapidly into the water coagulation bath. However, relatively little water diffuses into the casting solution since it is nonsolvent for an EVAL copolymer. Consequently, the surface casting solution enters the solidification region directly to form a dense top layer.

The diffusion of DMSO and water is very constrained by the dense top layer. As a result, only some water molecules diffuse into the interior portion of the casting solution to initiate some nuclei. These pores may grow rapidly by much DMSO diffusing into pores with a large driving force for DMSO and water. Thus finger-like cavities are clearly observed in membrane 1 .

As was discussed in the Theory section, the tendency for a change from a finger-like to a sponge-like structure membrane increases with decreasing mutual affinity of solvent and nonsol- vent when all parameters are kept constant. The outcome is the same as the addition of solvent into the coagulation bath when solvent and nonsolvent have a strong interaction [3,12,21], which reduces the interaction between the solvent and nonsolvent mixture. As Fig. 8 shows, the structure of membrane 2 using DMSO and water as the nonsolvent mixture (DMSO:water $=2: 1$ ) shows a vast difference from that of membrane 1 . This is because adding DMSO solvent into the coagulation bath reduces the interaction between the solvent and nunsoivent mixiure. Hence the diffifusion tendency of solvent into the coagulation is reduced. The surface casting solution thus crosses the binodal boundary to begin the liquid-liquid phase separation, and the result is a porous top layer. For water to enter the porous top layer, it can freely diffuse to initiate a the liquid-liquid phase separation. Since the many nuclei mutually limited their growth, the resulting membrane is porous with a sponge-like structure. Furthermore, because of the presence of DMSO in the coagulation bath, the interaction between the solvent and nonsolvent mixture is decreased. Therefore, the growth rate of pores will be decreased, and membrane 2 will not produce finger-like cavities.

Membrane 3 is the example using the above nonsolvent mixture (DMSO:water $=2: 1$ ) as the first coagulation bath (the residence time is $1 \mathrm{~s}$ ) followed by immersion in the water bath to fulfill 

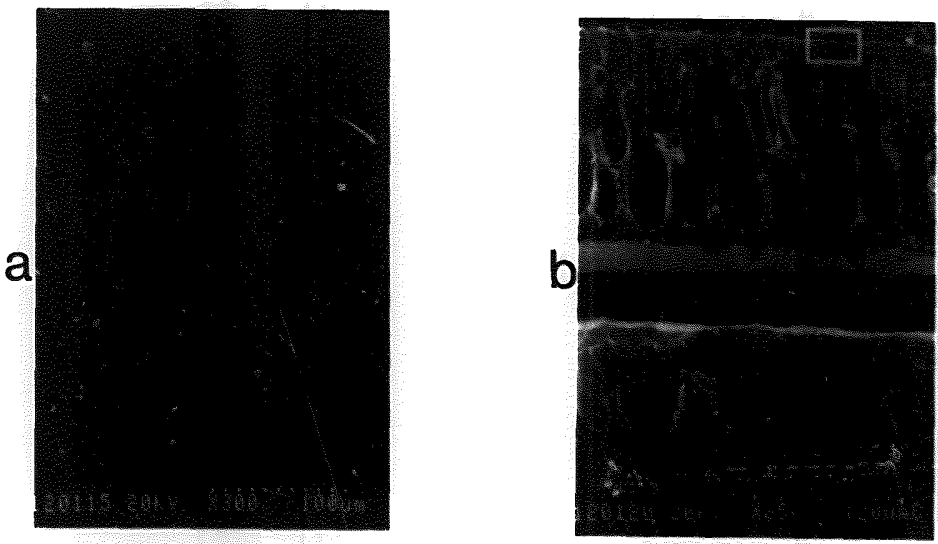

Fig. 7. SEM pictures of membrane 1. A, top; B, cross section.
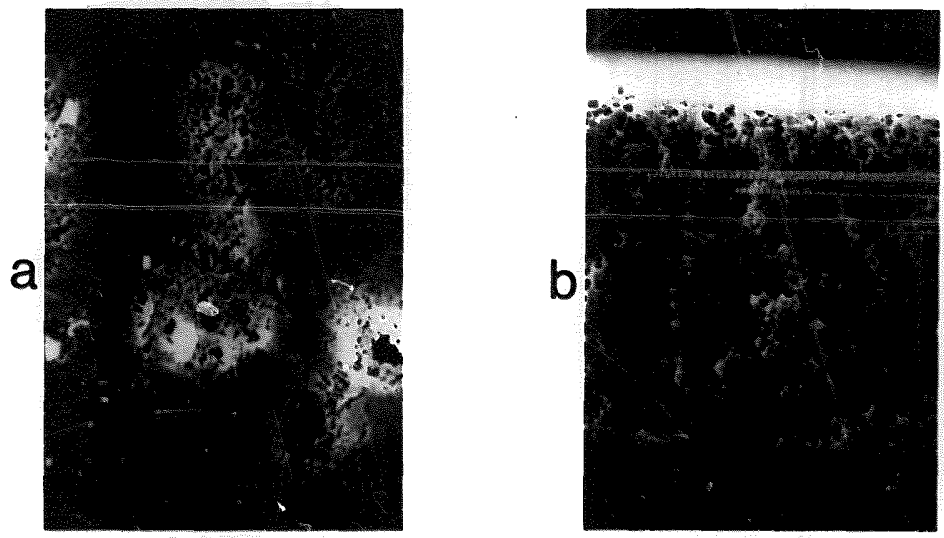

Fig. 8. SEM pictures of membrane 2. A, top; B, cross section.

the whole membrane. Since the casting solution initially coagulating in the first bath is not long enough to form the whole membrane, the first bath only produces the top layer. As Fig. 9A shows, membrane 3 can have the porous top layer due to the same coagulation condition as that of membrane 2 . This procedure produces pores in the top layer of membrane 1 before being immersed 

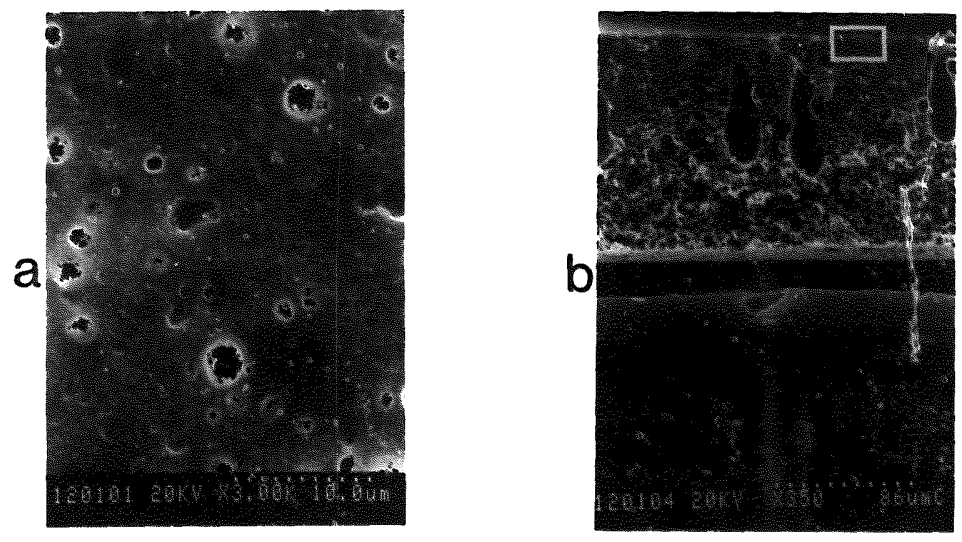

Fig. 9. SEM pictures of membrane 3. A. top; B, cress section.

directly into the coagulation bath consisting of water only. However, the residence time is too short to form the top layer completely, so the porosity of the top layer of membrane 3 is lower than that of membrane 2. Applying the principles, the porosity of the top layer formed in the first bath can be regulated.

The sublayer of membrane 3 is made by using water as a nonsolvent of the second coagulation bath. Water can diffuse through the porous top layer so that the sublayer forms more nuclei than membrane 1. Therefore, the growth of pores is constrained by other surrounding pores to form the uncompleted finger structure shown in Fig. 9B. In other words, the structure of membrane 3 is intermediate between the structure of membranes 1 and 2 .

\subsection{Membrane 4, 5}

The addition of nonsolvent into the casting solution has a considerable effect on the membrane structure. On adding water to the casting solution, DMSO can also leave the surface casting solution to form a dense skin. However, the initial compo-

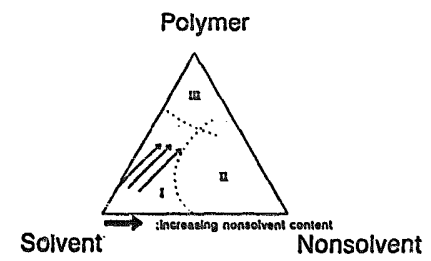

Fig. 10. Composition paths with varying nonsolvent content in the casting solution.

sition of casting solution moves closer to the region of the liquid-liquid phase separation in the ternary phase diagram. As discussed in the previous paper [13], the composition path of every layer in the ternary phase diagram can be considered as a straight line. Hence, the phase transition of the top layer may occur at a lower polymer concentration of solidification or even at liquidliquid phase separation, as shown in Fig. 10. Thus less dense structure or pores can be obtained. Fig. 11 shows that some pores occur in the top layer of Membrane 4, but it also has a skin structure. 

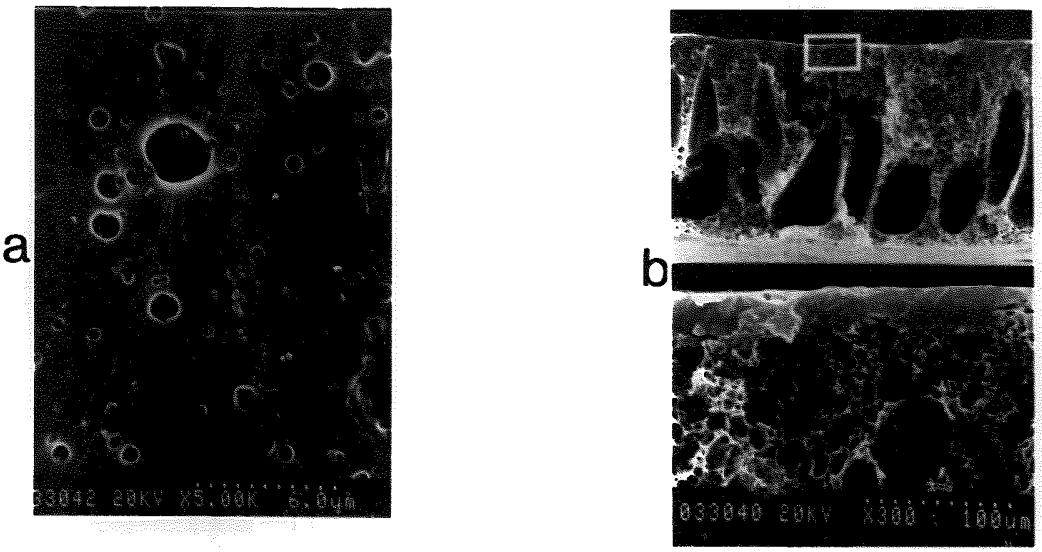

Fig. 11. SEM pictures of membrane 4. A, top; B, cross section.
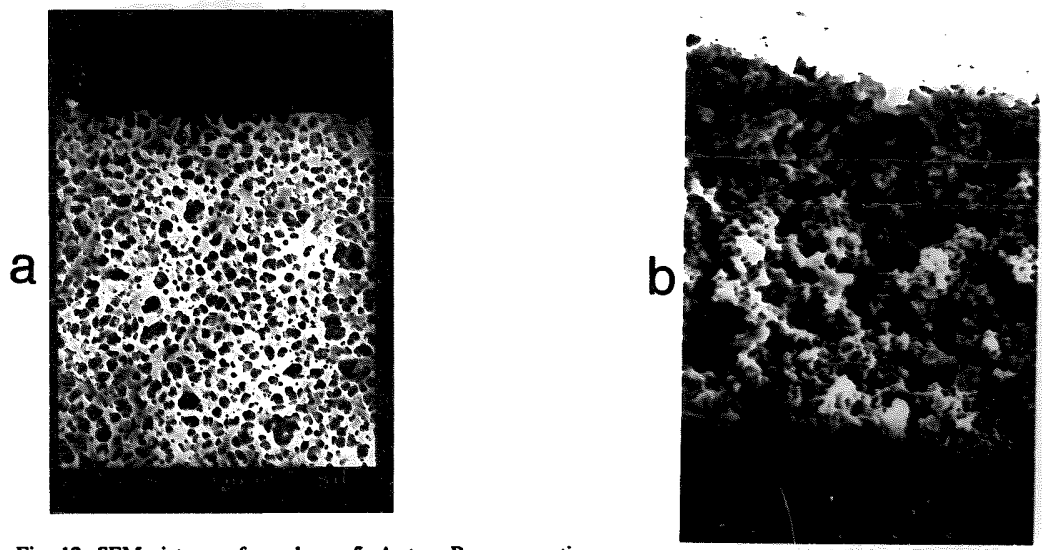

Fig. 12. SEM pictures of membrane 5. A, top; B, cross section.

From Fig. 11B, the structure of membrane 4 can be considered to consist of three parts: a dense top layer, a sponge-like structure and a finger-like structure. This results in the liquid-liquid phase separation in miscible polymer solution being brought about by variation in temperature and composition. A ternary polymer mixture can be a homogeneous casting solution if its composition is situated outside the liquid-liquid phase separation region. When the system is cooled, the liquid- 
liquid phase separation region will expand its range [11], and the location of the composition of the ternary system may be inside the liquid-liquid phase separation region to cause phase transition. Hence, when the top layer of membrane 4 was formed, the upper part of the sublayer was quickly cooled to shift its composition in the enlarging range of the demixing gap in the $25^{\circ} \mathrm{C}$ water coagulation bath. Because of the presence of large amounts of nonsolvent, many nuclei can be formed to inhibit the growth of the finger structure, thus membrane 4 cannot form finger-like structures in the upper part of the sublayer. However, due to a distance between the cool congulation bath and the lower solution, the temperatire of the lower soiution may not be decreased so rapidly that a homogeneous state may exist still. As a result, when a nonsolvent diffuses into the lower solution, some nuclei occur and grow into finger-like voids.

The example above does illustrate the principles of phase transition of the sublayer of membrane 4 for different reasons. The phase transition of the upper sublayer is caused by the presence of nonsolvent with decreasing temperature, which leads the upper solution to form many nuclei at the same time and to inhibit the formation of the finger-like structure. The phase transition of the lower sublayer is caused by nonsolvent diffusing into the region to form some nuclei locally. Then the nuclei grow to the finger-like voids.

If a $60^{\circ} \mathrm{C}$ ternary casting solution of membrane 4 cast on a glass plate is kept at $25^{\circ} \mathrm{C}$ for $5 \mathrm{~min}$ instead of being directly immersed into the coagulation bath, then the whole casting solution will induce the liquid-liquid phase separation by lowering temperature. In such a case many nuclei are formed by cooling the system to cause phase separation before a nonsolvent enters the casting solution. Therefore, membrane 5 does not have the finger-type structure shown in Fig. 12.

\section{Membrane 6, 7, 8}

As can be seen in Fig. 13, membrane 6 using two nonsolvents isopropanol alcohol (IPA) and water as a cosolvent for an EVAL copolymer has an increase in the pore numbers of the top layer. The solvation power of the cosolvent is much less than DMSO, so the homogeneous solution region greatly decreases. Hence, the top layer can precede the liquid-liquid phase separation immediately, resulting in many pores in the top layer of membrane 6. The top layer is porous enough to lead the large amount of nonsolvent into the sublayer to initiate many nuclei formations, so these nuclei limit each other's growth. Therefore, a porous structure and only smaller fingers are beneath the top layer in the cross section. However, it happens that the large pores in the lower part, which might be attributed to some nonsolvents, quickly diffuse to induce liquid-liquid phase separation before the solvent of the lower part diffuses out. Thus solvent can flow into the nascent pores to form large pores.

Membrane 7 was prepared with a casting solution at $70^{\circ} \mathrm{C}$; a 10 s evaporation period at $70^{\circ} \mathrm{C}$ shows a dense top layer (Fig. 14). At such a high temperature the polymer aggregates to develop a dense skin during the evaporation period [11]. Hence, when the casting solution is immersed into the coagulation bath, the dense skin offers a mass transfer resistance to both the diffusion rates of nonsolvent into the casting solution and solvent into the coagulation. Thus membrane 7 has fewer nuclei than membrane 6 and forms the larger pores, especially starting in the upper part of the sublayer.

Fig. 15 shows membrane 8 prepared from a $0^{\circ} \mathrm{C}$ coagulation bath that also has a skin layer on the top surface. This skin layer is believed to be primarily responsible for the very low temperature of the coagulation bath. The surface of the casting solution adjacent to the low temperature environment freezes immediately; thus polymer molecules solidify to result in a dense top layer. The sublayer solution and the $0^{\circ} \mathrm{C}$ coagulation bath are separated by the dense top layer, so the sublayer solution does not solidify quickly, and only a small amount of nonsolvent can diffuse through the top layer. 

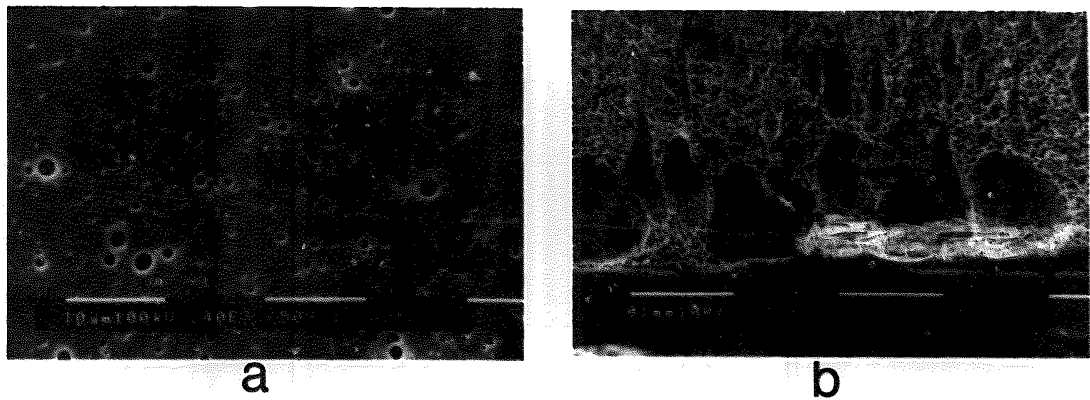

b

Fig. 13. SEM pictures of membrane 6. A, top; B, cross section.

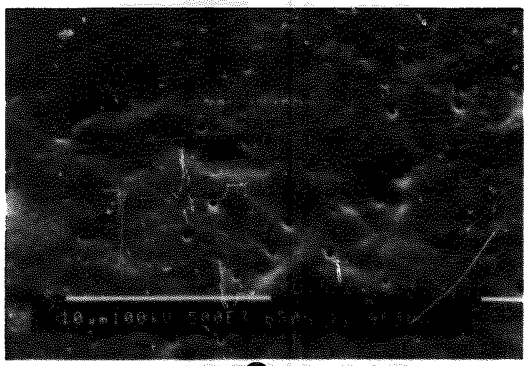

a
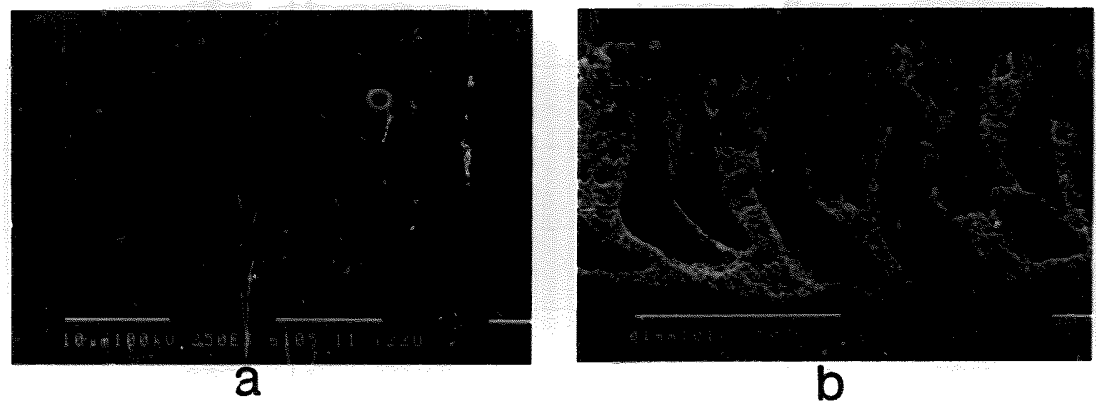

b

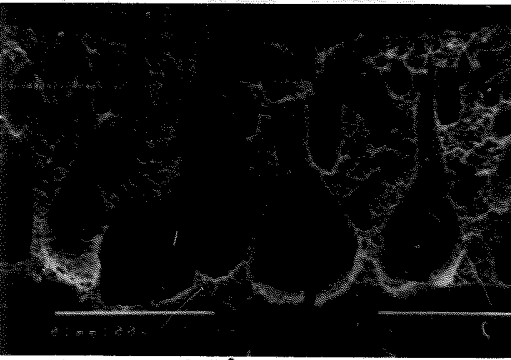

b

Fig. 15. SEM pictures of membrane 8. A, top; B, cross section.

Fig. 15. SEM pictures of membrane 8. A, top, B, cross section. 
Therefore, the large pores start very close to the top layer, and the finger-like structure is complete, as seen in Fig. 15B.

\section{Conclusions}

It has been pointed out that the formation of a finger-like structure can be divided into two steps: initiation and growth [4]. The formation mechanism of pores of this study provides a more detailed explanation. The initiation step produces nuclei by adding nonsolvent into the casting solution or by lowering the temperature of the casting solution. This is the point at which the content of nonsolvent has increased to the border of the binodal to form the initial nucleus, i.e., the polymer-poor phase from liquid-liquid phase separation. Once a nucleus has been initiated, the nucleus is capable of inducing solvent into it to grow pores according to the driving force between the solvent and nonsolvent. If the driving force is small, growth will be restricted. On the other hand, if the driving force is large enough, growth continues until the supply of solvent has been exhausted. However, if too many pores grow at the same time, they prevent themselves from further growth. Therefore, the pores in the membrane are the mature nuclei of the polymer-poor phase.

In applying the two-step mechanism model during membrane formation, one simply replaces the continuous variation of casting solution on top layer and sublayer regions. The top layer structure actually depends on the ratio of nonsolvent inflow and solvent outflow [12] (i.e., the composition path of the top layer enters the solidification or two-phase region). However, the top layer structure plays an important role in the initiation step for the structure of the sublayer. If the top layer structure is dense, the nonsolvent diffusion rate through the top layer is hindered and thus only some nuclei are formed. If the top layer structure is porous, much nonsolvent can diffuse into the sublayer to induce many nuclei at the same time. Hence, the surface structure determines the number of nuclei of the sublayer solution.
One can therefore conclude that membrane structure is determined by the relative diffusion rate of solvent and nonsolvent and the driving force between solvent and nonsolvent. Under conditions in which the out-diffusion rate of solvent is much faster than the in-diffusion rate of nonsolvent, the top layer is very dense, thus lowering the diffusion rate for nonsolvent into the sublayer. This results in fewer nuclei in the sublayer in the initial period. It is therefore possible to form a finger-like structure if the driving force between solvent and nonsolvent is strong enough. On the other hand, if the top layer is porous or the affinity between solvent and nonsolvent is low, the membrane only forms a sponge-like structure.

\section{Acknowledgment}

The authors thank the National Science Council of the Republic of China for their financial support, project NSC 83-0405-E-002-118T.

\section{References}

[1] S. Loeb and S. Sourirajan, Adv. Chem. Ser., 38 (1963) 117.

[2] R.E. Kesting, Synthetic Polymeric Membranes, Wiley, New York, 1985.

[3] H. Strathmann, K. Kock and P. Amar, Desalination, 16 (1975) 179.

[4] H. Strathmann and K. Kock, Desalination, 21 (1977) 241.

[5] L. Brones, D.M. Koenhen and C.A. Smolders, Desalination, 22 (1977) 205.

[6] D.M Koenhen, M.H.V. Mulder and C.A. Smolders, J. Appl. Polym. Sci., 21 (1977) 199.

[7] L. Brones, F.W. Altena and C.A. Smolders, Desalination, 32 (1980) 33.

[8] C.W. Yao, R.P. Burford, A.G. Fane and C.J.D. Fell, J. Membr. Sci., 38 (1988) 113.

[9] T. Uragami, Y. Ohsumi and M. Sugihara, Polymer, 23 (1982) 999.

[10] S. Munari, A. Bottino, G.C. Roda and G. Capannelli, Desalination, 77 (1990) 85.

[11] L.-W. Chen and T.-H. Young, Makromol., Chem. Macromol. Symp., 33 (1990) 183.

[12] T.-H. Young and L.-W. Chen, J. Membr. Sci., 57 (1991) 69. 
[13] T.-H. Young and L.-W. Chen, J. Membr. Sci., 59 (1991) 169.

[14] L.-W. Chen and T.-H. Young, J. Membr. Sci., 59 (1991) 15.

[15] T.-H. Young, Biomedical Engineering: Applications, Basis, Communication, 4 (1992) 71.

[16] L.C. Liu, Biomedical Engineering: Applications, Basis, Communication, 4 (1992) 365.

[17] T.-H. Young and L.-W. Chen, J. Membr. Sci., 83 (1993) 153.

[18] F.W. Altena and C.A. Smolders, Macromolecules, 15 (1982) 1491.

[19] L. Yimaz and A.J. McHugh, J. Appl. Polym. Sci., 31 (1986) 997.
[20] C. Cohen, G.B. Tanny and S.J. Prager, J. Polym. Sci., A-2, 17 (1979) 477.

[21] J.G. Wijmans, J.P.B. Baaij and C.A. Smolders, J. Membr. Sci., 14 (1983) 263.

[22] R.J. Ray, W.B. Krantz and R.L. Sani, J. Membr. Sci., 23 (1985) 155.

[23] L. Yilmaz and McHugh, J. Membr. Sci., 28 (1986) 287.

[24] A.J. Reuvers, J.W.A. van der Berg and C.A. Smo: ders, J. Membr. Sci., 34 (198i) 45.

[25] P. Radov, S.W. Thiel and S.-T. Hwang, J. Miention. Sci., 65 (1992) 213. 